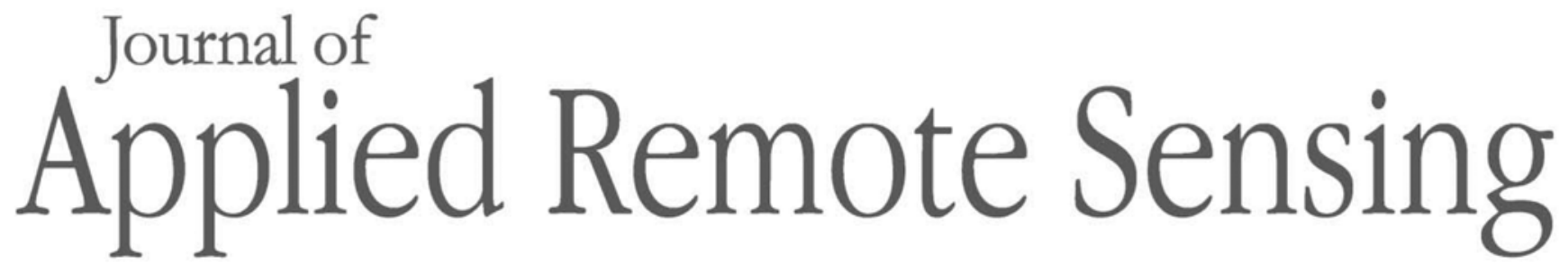

RemoteSensing.SPIEDigitalLibrary.org

\title{
Target deception jamming method against spaceborne synthetic aperture radar using electromagnetic scattering
}

Qingyang Sun

Ting Shu

Bin Tang

Wenxian $\mathrm{Yu}$ 


\title{
Target deception jamming method against spaceborne synthetic aperture radar using electromagnetic scattering
}

\author{
Qingyang Sun, Ting Shu,* Bin Tang, and Wenxian Yu \\ Shanghai Jiao Tong University, School of Electronic Information and Electrical Engineering, \\ Shanghai Key Laboratory of Intelligent Sensing and Recognition, Shanghai, China
}

\begin{abstract}
A method is proposed to perform target deception jamming against spaceborne synthetic aperture radar. Compared with the traditional jamming methods using deception templates to cover the target or region of interest, the proposed method aims to generate a verisimilar deceptive target in various attitude with high fidelity using the electromagnetic (EM) scattering. Based on the geometrical model for target deception jamming, the EM scattering data from the deceptive target was first simulated by applying an EM calculation software. Then, the proposed jamming frequency response (JFR) is calculated offline by further processing. Finally, the deception jamming is achieved in real time by a multiplication between the proposed JFR and the spectrum of intercepted radar signals. The practical implementation is presented. The simulation results prove the validity of the proposed method. (C) The Authors. Published by SPIE under a Creative Commons Attribution 3.0 Unported License. Distribution or reproduction of this work in whole or in part requires full attribution of the original publication, including its DOI. [DOI: 10.1117/1.JRS.12.016033]
\end{abstract}

Keywords: synthetic aperture radar; spaceborne synthetic aperture radar; deceptive jamming; electromagnetic scattering.

Paper 170860 received Sep. 30, 2017; accepted for publication Feb. 16, 2018; published online Mar. 7, 2018.

\section{Introduction}

Synthetic aperture radar (SAR) has been widely applied in both civil surveillance and military reconnaissance. It is capable of generating high-resolution images in all weather conditions and, thus, can provide a powerful tool for remote sensing applications, such as target detection, recognition, and classification. ${ }^{1-3}$ In addition, to protect the information of targets from being observed by the hostile forces, the electronic countermeasures (ECM) techniques against SAR have also been developed rapidly during the past decades. ${ }^{4}$

Generally, the ECM techniques against SAR include the barrage jamming and the deception jamming. In barrage jamming, the jammer transmits the noise-like jamming signals into SAR echoes with a very high jamming power, which may be easy to be suppressed and not be applicable in practice. ${ }^{5,6}$ In the direct retransmitting deception jamming, ${ }^{7-9}$ the jammer generates the coherent jamming signals by a convolution between the unit impulse response of the jammer system and the intercepted radar signals with a much lower transmitting power than the barrage jamming.

Zhou et al. ${ }^{7}$ proposed a large scene deception jamming method and discussed the real-time realization using a two-step realization and subtemplates parallel processing. Liu et al. ${ }^{8}$ presented an efficient frequency-domain three-stage algorithm for deception jamming against SAR using the fast Fourier transform (FFT) to accelerate the implementation of the method. Sun et al. ${ }^{9}$ also proposed an efficient deception jamming technique against SAR by separating the modulation process of the jammer into offline stage and real-time stage, which is easy to be implemented in practical real-time application. Pace et al. ${ }^{10}$ introduced an all-digital image synthesizer capable of generating false target images from a series of intercepted chirp pulses for countering inverse

*Address all correspondence to: Ting Shu, E-mail: tingshu@sjtu.edu.cn 
SAR (ISAR), while a deception template of a ship that closely matches an actual ISAR image is also utilized. In summary, the main purpose of these methods is to cover the region of interest using the deception templates, which are usually derived from an existing SAR image. However, the false target generated by these methods is difficult to be able to include the similar structural or scattering information being relative to a hostile SAR system. Therefore, since the false target is limited to the deception templates, the scattering characteristic of the false target is not applicable when the false target should be changed to another look angle without distortion.

In this paper, we propose a method to perform target deception jamming against spaceborne SAR in this paper using the electromagnetic (EM) scattering. Compared with the traditional jamming methods using deception templates, the proposed method achieves the deception jamming using the EM scattering from a deceptive target, which is able to generate the deceptive target in various attitude with high fidelity. Based on the geometrical model for target deception jamming, the EM scattering data from the deceptive target are first simulated by applying an EM calculation software, such as the Computer Simulation Technology (CST) Microwave Studio. ${ }^{11}$ Then, the proposed jamming frequency response (JFR) is calculated offline by further processing. Finally, the deception jamming is achieved in real time by a multiplication between the JFR and the spectrum of intercepted radar signals.

The rest of this paper is organized as follows. The principle for target deception jamming is discussed in Sec. 2. The practical implementation is discussed in Sec. 3. Section 4 shows the deception jamming simulation results. Section 5 concludes this paper.

\section{Principle for Target Deception Jamming}

\subsection{Basic Principle for Target Deception Jamming}

Figure 1 shows the geometrical model for target deception jamming against spaceborne SAR in the two-dimensional (2-D) slant range plane, including the radar coordinates system $r O x$ and the target deception coordinates system $u C v$.

Suppose that the spacebrone SAR to be interfered works at the broadside and strip-map mode, and moves at a speed of $V_{r}$ along the $x$-axis. Use $t_{a}$ to represent the slow time, and the zero moment of $t_{a}$ is set at the moment when the SAR is located at $O$. As shown in Fig. 1, the jammer and the center of target deception coordinates system are located at $\left(R_{J}, x_{J}\right)$ and $\left(R_{c}, 0\right)$ in the radar coordinates system, respectively. The instantaneous position of SAR is $(0, x)$ or $\left(0, V_{r} t_{a}\right)$ in the radar coordinates system; $L_{\mathrm{sar}}$ is the synthetic aperture length. $(u, v)$ and $\sigma(u, v)$ are the location and complex scattering coefficients of a point scatterer in the deceptive target, respectively. $R_{c}\left(t_{a}\right)$ is the instantaneous slant range between the radar and

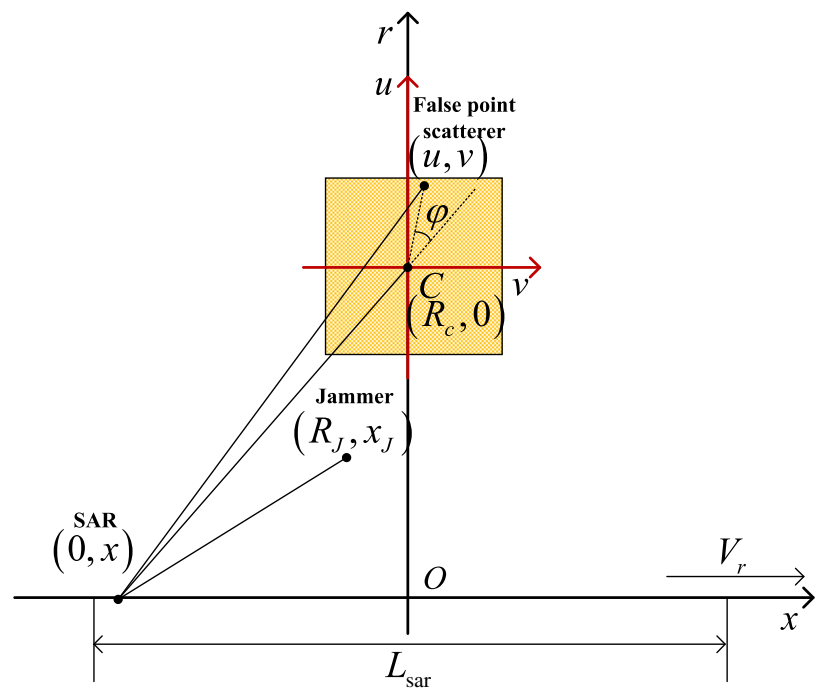

Fig. 1 The geometrical model for target deception jamming. 
the center of deception coordinates system. $R_{J}\left(t_{a}\right)$ is the instantaneous slant range between the radar and the jammer. $R\left(t_{a}, u, v\right)$ is the instantaneous slant range between the radar and a point scatterer in the deceptive target.

Without loss of generality, we consider that the spaceborne SAR transmits a chirp signal. Then, a jammer can be seen as a special point scatterer in the illuminated scene by the SAR, whose baseband received echoes ${ }^{1}$ can be expressed as

$$
s_{J}\left(\tau, t_{a}\right)=a\left[\tau-\frac{2 R_{J}\left(t_{a}\right)}{c}\right] \exp \left[-j 2 \pi f_{c} \frac{2 R_{J}\left(t_{a}\right)}{c}\right] \exp \left\{j \pi K_{r}\left[\tau-\frac{2 R_{J}\left(t_{a}\right)}{c}\right]^{2}\right\},
$$

where $\tau$ is the fast time, $a(\tau)=\operatorname{rect}\left(\frac{\tau}{T_{p}}\right)$ yields 1 when $\left|\frac{\tau}{T_{p}}\right|<0.5$ and is 0 otherwise, $T_{p}$ is the pulsewidth, $f_{c}$ is the carrier frequency, $K_{r}$ is the chirp rate, and $c$ is the speed of light.

If the deceptive target existed, its echo can be given by

$$
\begin{aligned}
s\left(\tau, t_{a}\right)= & \iint \mathrm{d} u \mathrm{~d} v \sigma(u, v) a\left[\tau-\frac{2 R\left(t_{a}, u, v\right)}{c}\right] \times \exp \left[-j 2 \pi f_{c} \frac{2 R\left(t_{a}, u, v\right)}{c}\right] \\
& \times \exp \left\{j \pi K_{r}\left[\tau-\frac{2 R\left(t_{a}, u, v\right)}{c}\right]^{2}\right\},
\end{aligned}
$$

where $\sigma(u, v)$ is the backscattering coefficient of a point scatterer in the deceptive target, which is located at $(u, v)$ in the target deception coordinates system.

According to the principle of deception jamming against $\mathrm{SAR},{ }^{8}$ the retransmitted jamming signal $s\left(\tau, t_{a}\right)$ for the deceptive target of a jammer can be considered as a result of a convolution between the intercepted radar signal $s_{J}\left(\tau, t_{a}\right)$ and the unit impulse response $h\left(\tau, t_{a}\right)$ of the jammer system, where $h\left(\tau, t_{a}\right)$ satisfies $s\left(\tau, t_{a}\right)=s_{J}\left(\tau, t_{a}\right) \otimes h\left(\tau, t_{a}\right)$ and $\otimes$ denotes the convolution operation along the fast time $\tau$.

Because it is more efficient to implement the convolution operation in frequency domain, we perform the FFT to $h\left(\tau, t_{a}\right)$ along the fast time $\tau$ yields

$$
H\left(f_{\tau}, t_{a}\right)=\iint \mathrm{d} u \mathrm{~d} v \sigma(u, v) \exp \left[-j \frac{4 \pi\left(f_{c}+f_{\tau}\right)}{c} \Delta R\left(t_{a}, u, v\right)\right],
$$

with

$$
\Delta R\left(t_{a}, u, v\right)=R\left(t_{a}, u, v\right)-R_{J}\left(t_{a}\right)
$$

where $H\left(f_{\tau}, t_{a}\right)$ is the alleged JFR.

\subsection{Proposed Method}

Generally, a jammer aims to plant the jamming signals of deception templates into the raw echoes of a real scene. However, the backscattering coefficients of the deception templates are usually derived from an existing SAR image, which is chosen according to the scattering characteristics of the SAR images to be interfered. On one hand, it can hardly include the similar structural or scattering information being relative to a hostile SAR system. On the other hand, the scattering characteristic of the false target is also limited to the deception templates so that the deceptive target may not be changed to another required attitude angle without distortion.

Thus, it is necessary to combine the EM scattering from the target with the deception jamming to generate the verisimilar deceptive target with high fidelity in various attitude.

According to Fig. 1, $R_{c}\left(t_{a}\right)$ and $R_{J}\left(t_{a}\right)$ can be represented as Eqs. (5) and (6), respectively.

$$
\begin{gathered}
R_{c}\left(t_{a}\right)=\sqrt{R_{c}^{2}+V_{r}^{2} t_{a}^{2}}, \\
R_{J}\left(t_{a}\right)=\sqrt{R_{J}^{2}+\left(V_{r} t_{a}-x_{J}\right)^{2}} .
\end{gathered}
$$


Meanwhile, $R\left(t_{a}, u, v\right)$ can be expressed as

$$
R\left(t_{a}, u, v\right)=\sqrt{R_{1}\left(t_{a}, u, v\right)^{2}+R_{2}\left(t_{a}, u, v\right)^{2}},
$$

with

$$
\begin{gathered}
R_{1}\left(t_{a}, u, v\right)=\sqrt{R_{c}^{2}+V_{r}^{2} t_{a}^{2}}+\sqrt{u^{2}+v^{2}} \cos \left[\arctan \left(\frac{-V_{r}}{R_{c}} t_{a}\right)-\arctan \left(\frac{v}{u}\right)\right] \\
R_{2}\left(t_{a}, u, v\right)=\sqrt{u^{2}+v^{2}} \sin \left[\arctan \left(\frac{-V_{r}}{R_{c}} t_{a}\right)-\arctan \left(\frac{v}{u}\right)\right]
\end{gathered}
$$

When we fabricate the nonexistent small deceptive target against a typical spaceborne SAR platform, it is useful to make the approximation

$$
R\left(t_{a}, u, v\right) \approx R_{1}\left(t_{a}, u, v\right)=R_{c}\left(t_{a}\right)+p\left(u, v, t_{a}\right),
$$

Because the distance between the spaceborne SAR and the deceptive target is much larger than the size of the target. Moreover, $p\left(u, v, t_{a}\right)$ can also be seen as the projection of the vector directed from the center of the target deception coordinates system to the scatterer on the vector directed from the radar to the center of the target deception coordinates system.

According to Fig. 1, the projected look angle $\varphi$ can be denoted as

$$
\varphi=\arctan \left(\frac{-V_{r}}{R_{c}} t_{a}\right)-\arctan \left(\frac{v}{u}\right)
$$

where $\arctan \left(\frac{v}{u}\right)$ is the initial attitude angle of the target.

Substituting Eq. (10) into Eqs. (4) and (3), one obtains

$$
H\left(f_{\tau}, t_{a}\right)=\exp \left\{-j \frac{4 \pi\left(f_{\tau}+f_{c}\right)}{c}\left[R_{c}\left(t_{a}\right)-R_{J}\left(t_{a}\right)\right]\right\} \times A\left(f_{\tau}, t_{a}\right),
$$

with

$$
A\left(f_{\tau}, t_{a}\right)=\iint \sigma(u, v) \mathrm{d} u \mathrm{~d} v \exp \left[-j \frac{4 \pi\left(f_{\tau}+f_{c}\right)}{c} p\left(u, v, t_{a}\right)\right],
$$

where $A\left(f_{\tau}, t_{a}\right)$ is the frequency response of EM scattering from the deceptive target, and $H\left(f_{\tau}, t_{a}\right)$ in Eq. (12) is the proposed JFR.

Because the electronic warfare support measure can well estimate the transmitted signal parameters, such as the bandwidth and carrier frequency, and the spaceborne SAR kinematic parameters can also be predicted by the Kepler's laws according to the satellite orbit in advance, the proposed JFR can be calculated offline in practical application and stored in the digital radiofrequency memory (DRFM).

Compared with the deception jamming method using an existing SAR image as the deception template, the proposed method introduces the EM scattering property of the target into the generation of jamming signals. Therefore, since the calculated EM scattering property is related to the attitude of the target, it is able to generate the deceptive target in various attitude with high fidelity. In this paper, $A\left(f_{\tau}, t_{a}\right)$ can be simulated using an EM calculation tool, such as the CST Microwave Studio. ${ }^{11}$ To obtain EM scattering property, the frequency response of scattering from the false target is simulated using a stepped frequency waveform (SFW). The parameters of the SFW, such as range and spacing, are designed considering the bandwidth of the radar signal and the size of the target. Because it has to cover the frequency band and obtain the sufficient unambiguous range. Additionally, the scattering is influenced by the azimuth time. Different azimuth time corresponds to different projected look angles. Thus, it is simulated by setting a set of look angles in the CST. Then, the detailed EM scattering data from the deceptive target are calculated by utilizing the shooting and bouncing rays techniques, which is in the asymptotic solver of CST Microwave Studio. 


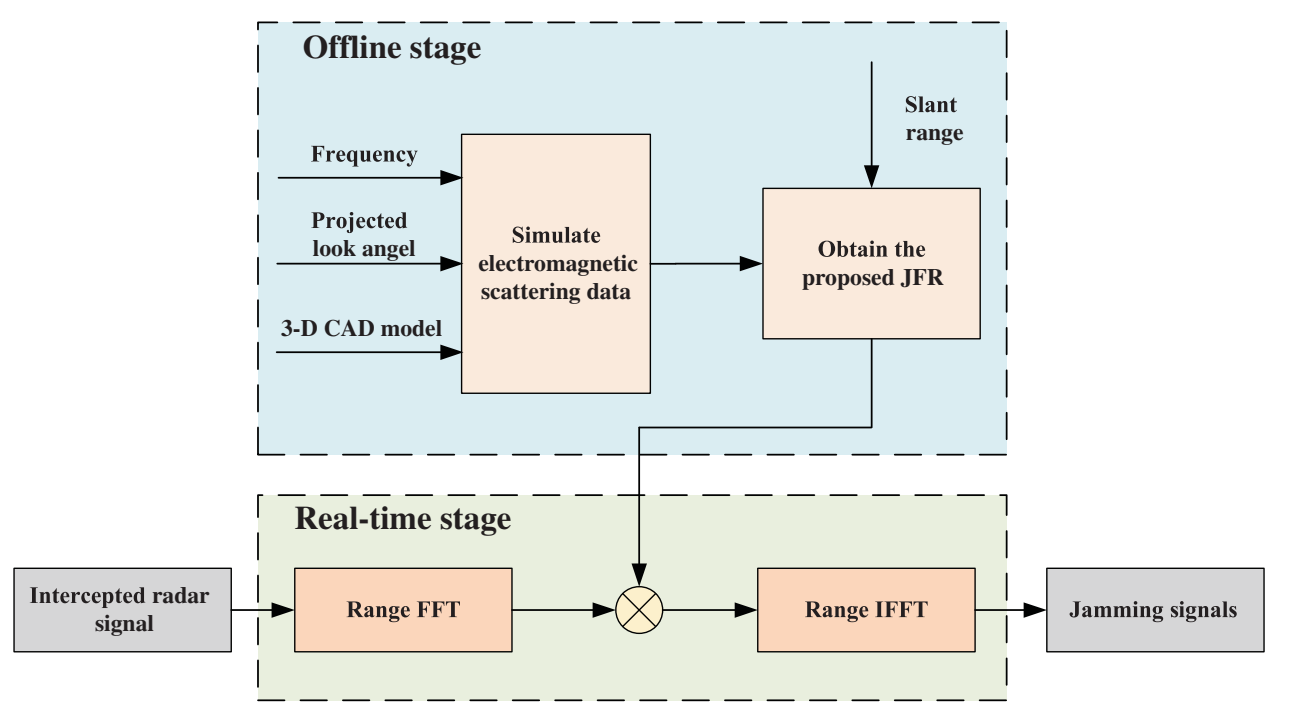

Fig. 2 The block diagram of the proposed method.

\subsection{Implementation of Algorithm}

Based on the above analysis, the block diagram of the proposed method is shown in Fig. 2.

Hence, the proposed deception jamming method can be achieved as follows:

1. Simulate the EM scattering from the three-dimensional (3-D) CAD model of the deceptive target using an EM calculation tool, such as the CST Microwave Studio.

2. Obtain the proposed JFR according to Eq. (12) and store them in the DRFM.

3. Implement a multiplication between the JFR stored in the DRFM and the spectrum of radar signals intercepted by the jammer and then retransmit the jamming signals to the spaceborne SAR system in range time domain by applying the range inverse FFT.

\section{Practical Implementation}

To capture the signal of interest, a very wide bandwidth of radio frequency (RF) front-end is used, typically 6 to $12 \mathrm{GHz}$. The received SAR signal is sent to a wideband RF splitter, and the two outputs of the RF splitter are sent to the instantaneous frequency measurement (IFM) receiver and the DRFM, respectively. A 6- to $12-\mathrm{GHz}$ IFM receiver is used to track the center frequency of the SAR signal with very small time delay. The accuracy of the IFM receiver is $<1 \mathrm{MHz}$ and is adequate for tracking the center frequency. Then, the frequency measurement results of the IFM receiver are sent to the frequency synthesizer to generate the changeable local oscillator (LO). This LO is common for both the downconversion mixer and the upconversion mixer. Thus, the coherency of the jammer signal is guaranteed. In other words, the carrier frequency of the RF output jammer signal is nearly the same as the SAR signal.

The operation of the wideband DRFM in Fig. 3 is as follows. The input SAR signal is downconverted by the common LO and then sampled with a high-speed analog-to-digital converter (ADC). The samples are stored in digital memory through a field programmable gate array (FPGA) for later retrieval. If the sample rate of ADC and the storage resources of FPGA are adequate, the parameters of the captured SAR signal (e.g., bandwidth, pulse width, amplitude, frequency, and phase) will be fully replicated. Moreover, the amplitude, frequency, and phase of the samples stored in digital memory can be manipulated to generate a wide range of jammer signals. The stored samples are later recalled, passed through a digital-to-analog converter, upconverted by the common LO, and transmitted back to the SAR system. To guarantee the coherency of the jammer signal, the bandwidth of DRFM should not be less than the bandwidth of the SAR signal.

The block diagram of a DRFM-based SAR jammer system is shown in Fig. 3. 


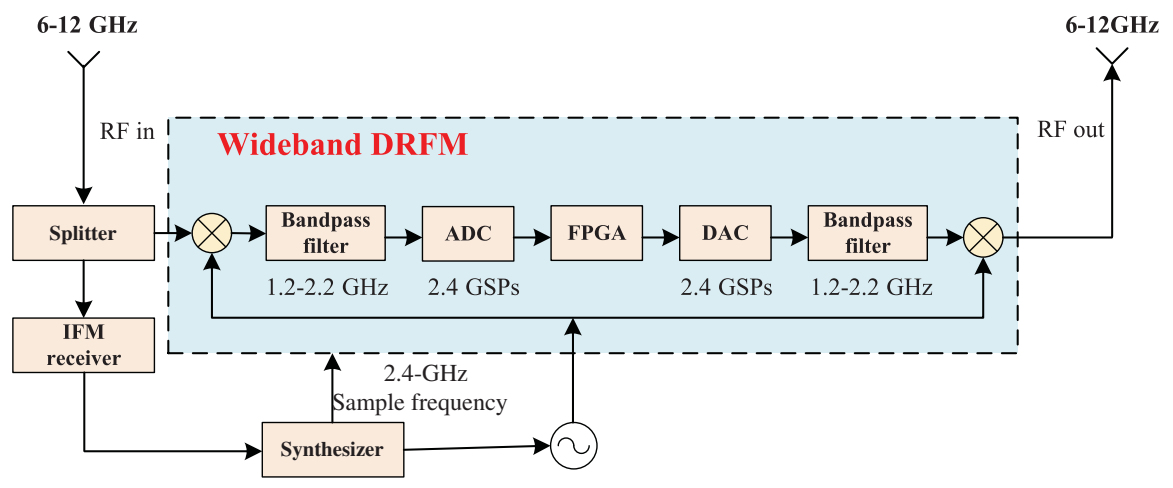

Fig. 3 Block diagram of DRFM-based SAR jammer system.

Take the example of the block diagram in Fig. 3, the jammer system has the capability of capturing and replicating the SAR signal with maximum instantaneous bandwidth of $1 \mathrm{GHz}$, using ADC with 2.4-GSPs sample rate and the DRFM with 1-GHz bandwidth. In previous work, an experimental jammer system has been built up and several hardware-in-the-loop experiments have been carried out. ${ }^{9}$

\section{Simulation Results}

In this section, some examples are presented to assess the validity of the proposed method. A typical X-band spaceborne SAR platform working in the broadside and strip-map mode is set up in the simulation. The system parameters are also listed in Table 1.

\subsection{Analysis of Focusing}

In the deception jamming against spaceborne SAR, the jammer intercepts the chirp signals transmitted by the radar and then retransmits jamming signals to the radar. Consequently, the imaging result along the range is well-focused as the transmitted chirp rate is not changed during the modulation procedure. Therefore, we mainly analyze the image focusing along azimuth.

In the experiment, the position of the center of the target deception coordinates system is located the same as that of the jammer, because the former mainly determines a relative location to the false target from the jammer and has no impact on the focusing capability. Moreover, five scatterers are set as the false targets for the analysis of focusing, which are located at

Table 1 Simulation system parameters.

\begin{tabular}{lcc}
\hline \hline Parameters & Values & Units \\
\hline Carrier frequency & 9.65 & $\mathrm{GHz}$ \\
Chirp bandwidth & 0.15 & $\mathrm{GHz}$ \\
Sampling frequency & 0.165 & $\mathrm{GHz}$ \\
Pulse repetition frequency & 4147.2 & $\mathrm{~Hz}$ \\
Velocity & 7113.59 & $\mathrm{~m} / \mathrm{s}$ \\
Squint angle & 0 & $\mathrm{deg}$ \\
Orbit altitude & 514.8 & $\mathrm{~km}$ \\
Resolution of azimuth & $\approx 3$ & $\mathrm{~m}$ \\
Resolution of range & $\approx 1$ & $\mathrm{~m}$ \\
\hline \hline
\end{tabular}




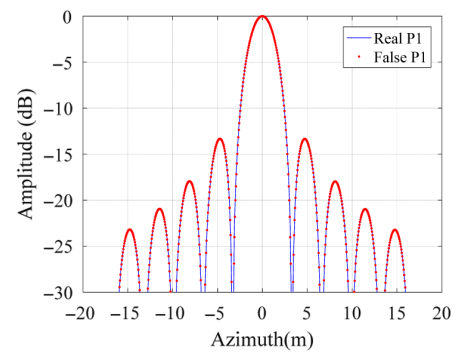

(a)

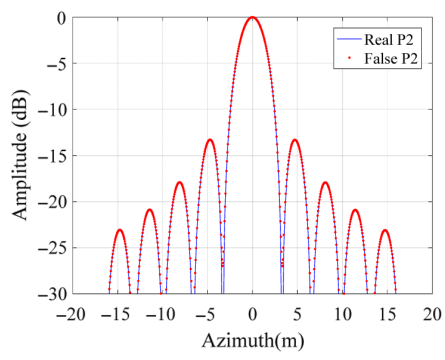

(b)

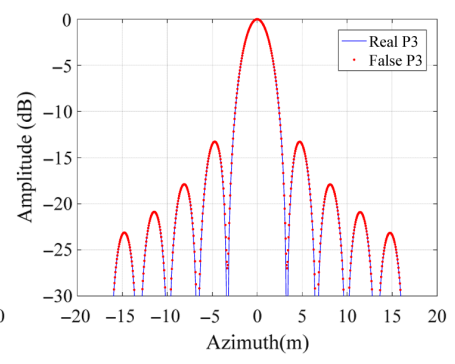

(c)

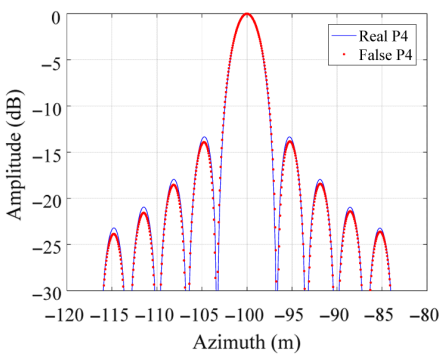

(d)

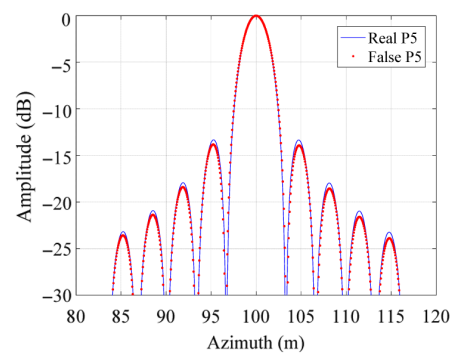

(e)

Fig. 4 PSF along azimuth of the five real and false scatterers: (a) P1, (b) P2, (c) P3, (d) P4, and (e) P5.

$(u, v)=(0,0),(-100 \mathrm{~m}, 0),(100 \mathrm{~m}, 0),(0,-100 \mathrm{~m})$, and $(0,100 \mathrm{~m})$ in the target deception coordinates system. The real scatterers are set at the same position as the false scatterers for contrast analysis. The scatterers are numbered as P1 to P5 in sequence.

The raw echoes of the five real scatterers are achieved by the method in Ref. 12. The jamming signals with the five isolated false scatterers are generated by the proposed method. The $\omega \mathrm{K}$ imaging algorithm is applied to obtain the SAR images. ${ }^{1}$ Then, the point spread functions (PSF) along azimuth of the five real and false scatterers are shown in Fig. 4. The solid lines and the dot lines denote the real scatterers and the false scatterers, respectively.

To demonstrate the validity of the proposed method quantitatively, the detailed evaluating results, including impulse response width (IRW), peak sidelobe ratio (PSLR), and integrated sidelobe ratio (ISLR), are listed in Table 2. The IRW is defined as the width of the main lobe of the impulse response, measured $3 \mathrm{~dB}$ below the peak value. The PSLR is the ratio between the height of the largest sidelobe and the height of the main lobe. The ISLR is the ratio between the total power of the sidelobes and the power of the main lobe. The PSLR and ISLR are both expressed in decibels $(\mathrm{dB})$. As shown in Table 2, it is accurate for P1, which is also the center of the target deception coordinates system, but not for the other four scatterers. And, we can see there is only minor difference in the IRW between the

Table 2 The detailed evaluating results.

\begin{tabular}{lccccc}
\hline \hline Scatterer & P1 & P2 & P3 & P4 & P5 \\
\hline IRW of real $(\mathrm{m})$ & 2.921 & 2.921 & 2.922 & 2.950 & 2.949 \\
IRW of false $(\mathrm{m})$ & 2.921 & 2.925 & 2.926 & 2.980 & 2.981 \\
IRW error $(\%)$ & 0.00 & 0.14 & 0.14 & 1.02 & 1.09 \\
PSLR of real $(\mathrm{dB})$ & -13.335 & -13.342 & -13.334 & -13.336 & -13.336 \\
PSLR of false $(\mathrm{dB})$ & -13.333 & -13.277 & -13.277 & -13.874 & -13.874 \\
ISLR of real $(\mathrm{dB})$ & -10.106 & -10.080 & -10.093 & -10.108 & -10.109 \\
ISLR of false $(\mathrm{dB})$ & -10.105 & -9.978 & -9.978 & -10.722 & -10.722 \\
\hline \hline
\end{tabular}


other four false scatterers and the corresponding real scatterers, according to Table 2, where the minor differences are caused by the approximation of range equation, as expressed in Eq. (10). However, the IRW errors are too small to cause an obvious defocusing effect in the jamming result along azimuth direction. Moreover, the image contrasts indicated by PSLR or ISLR of the false targets have nearly the same or a little better performance compared with those of the real targets. It means that the imaging quality of false targets obtained by the proposed method along azimuth is similar to that of real targets. In addition, it is also indicated that the approximation in Eq. (10) is acceptable and will not greatly affect the performance of the proposed method while fabricating the small target. This is because the distance to the target is long and the synthetic aperture duration is relatively short for spaceborne SAR. Therefore, the experiments verify that the proposed method can generate well-focused false scatterers, and the nonexistent small deceptive targets are expected to be well-focused under the proposed method.

\subsection{Deceptive Target Simulation}

The simulation parameters of the spaceborne SAR system are also listed in Table 1.

In this experiment, we aim to plant a deceptive target into the real scene. The real scene is shown in Fig. 5, which is near the coast and consists of $512 \times 512$ pixels. In the meantime, a typical destroyer is chosen as the deceptive target. The high precision 3-D CAD model of the destroyer is shown in Fig. 6, whose length and width are roughly equivalent to 161.8 and $20 \mathrm{~m}$, respectively. Generally, a deception template with a destroyer is pretty hard to be found in the

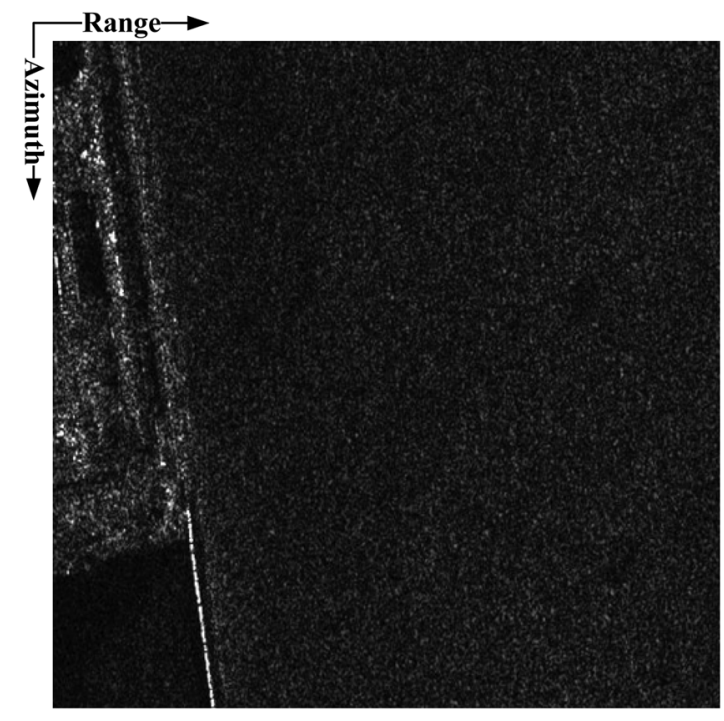

Fig. 5 The real scene with $512 \times 512$ pixels.

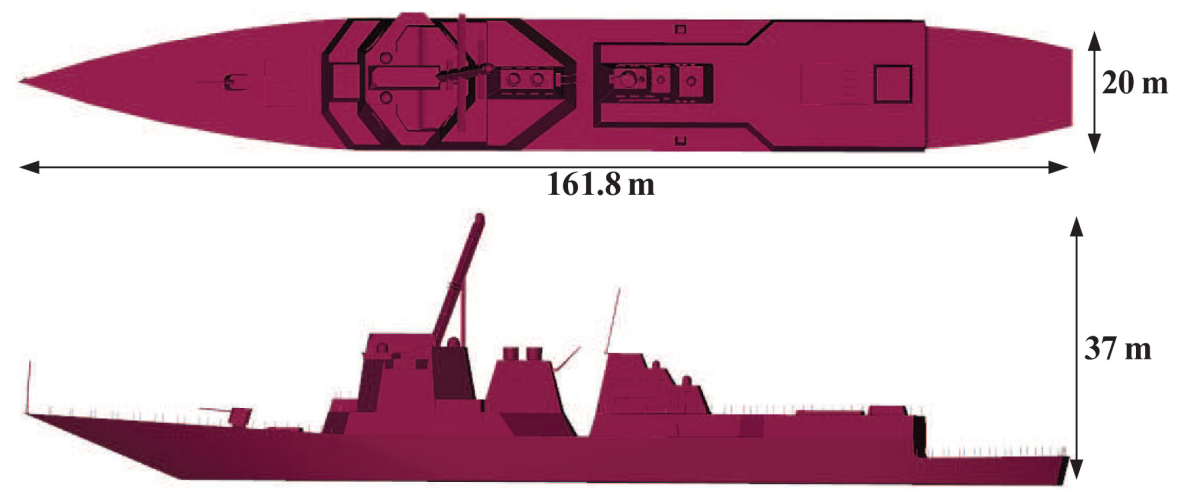

Fig. 6 The high precision CAD model of a typical destroyer. 


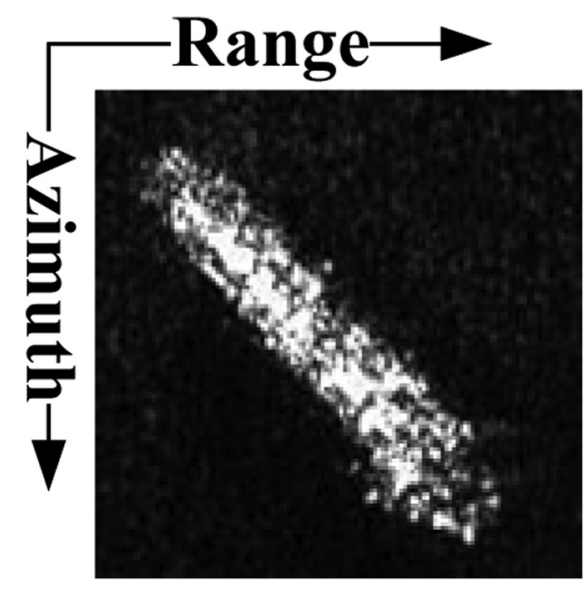

Fig. 7 A chip SAR image with a civilian ship.

existing SAR images. It leads to the traditional method based on the deception template can hardly generate the desired destroyer. Hence, the proposed method also contributes to the generation of the specific deceptive target.

In addition, another superiority is that the proposed method is able to generate the deceptive target in various attitude with high fidelity. To demonstrate the superiority of the proposed method, we perform a comparison with an existing deception template-based method in Ref. 7. On one hand, a chip SAR image is chosen as the deception template, in which a civilian ship is considered as the deceptive target, as shown in Fig. 7. In the SAR image, the length and width of the ship are $\sim 245.6$ and $36.93 \mathrm{~m}$, respectively. Then, the jamming result of the deceptive target in two different attitudes is performed by applying the method in Ref. 7. One of two attitudes is consistent with that of the original chip SAR image, and another is rotated 50-deg clockwise. On the other hand, as for the proposed deception jamming, the EM scattering data from the destroyer in two different attitudes are built up ahead of time by means of the CST Microwave Studio software. The attitude of the target is set in the CST Microwave Studio software. Different attitude of the target corresponds to different frequency response of EM scattering according to Eq. (13). When the offline calculation of frequency response of EM scattering is finished, the jamming signals of the deceptive target in various attitude are then generated using the proposed method. Similarly, the $\omega \mathrm{K}$ algorithm is applied to the image formation.

Figures 8 (a) and 8(b) show the imaging results after the deception jamming achieved by Ref. 7 in two different attitudes, respectively. They are numbered as DT1 to DT2 in sequence.

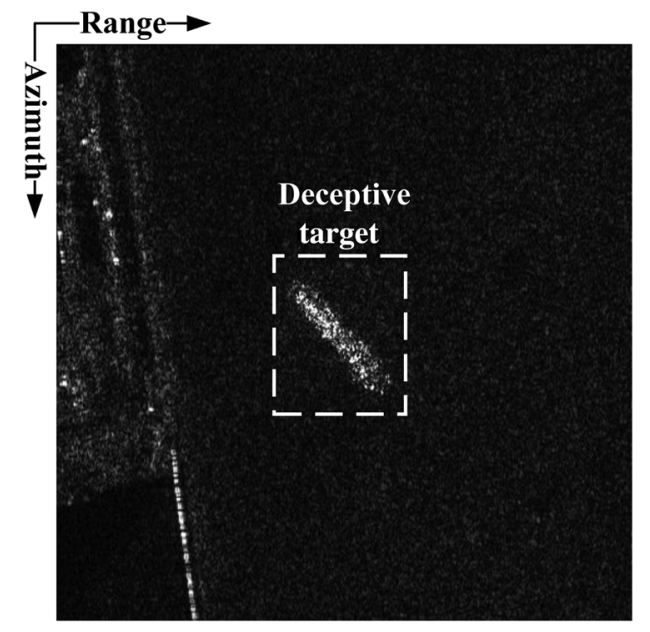

(a)

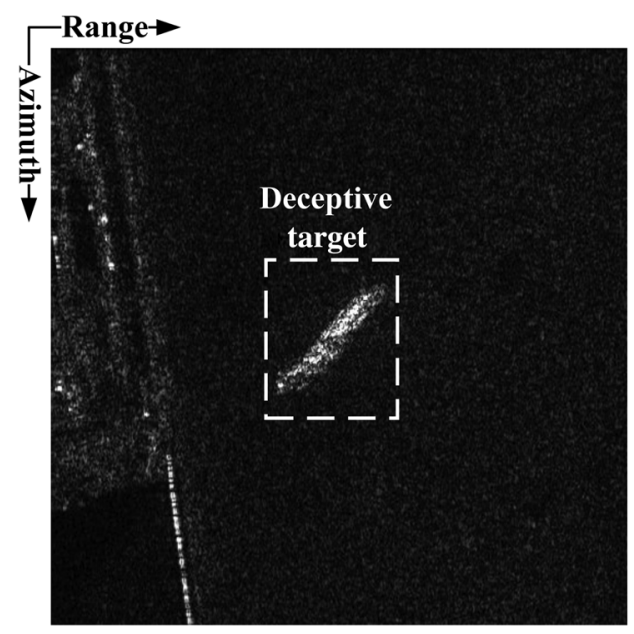

(b)

Fig. 8 The jamming result of Ref. 7 with $512 \times 512$ pixels: (a) DT1 and (b) DT2. 


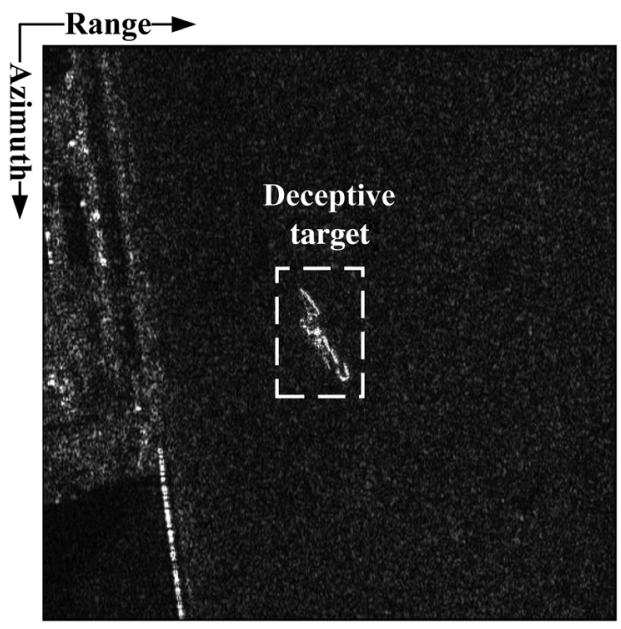

(a)

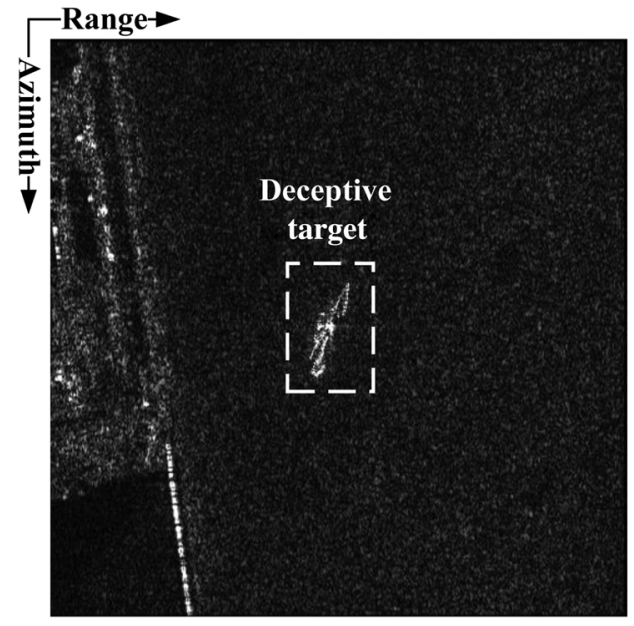

(b)

Fig. 9 The jamming results of the proposed method with $512 \times 512$ pixels: (a) DT3 and (b) DT4.

It is observed that the method in Ref. 7 is able to generate a well-focused deceptive target, as shown in Fig. 8(a), where the attitude is identical with that of the deception template. However, the performance of the deception jamming will start to deteriorate once the attitude of the target is changed to another one. Figure 8(b) shows the result of the deterioration as the contour of the ship is too distorted. On the other hand, the imaging results after deception jamming of the proposed method in two different attitudes are shown in Figs. 9(a) and 9(b), respectively. They are numbered as DT3 to DT4 in sequence. It is observed that the contour of the deceptive target in the two different attitudes is similar to that of the destroyer. In addition, the open rail of the destroyer in the two jamming results corresponds to the scattering effects of shadowing, because the warship's superstructure, such as the mainmast and bridge, occludes the radar signals partly illuminating the distant rail, in which it appears black. Moreover, it is seen that the well-focused deceptive target merges with the real scene, which makes the SAR hard to distinguish the false target. Generally, the geometrical feature is another widely explored ship feature used for ship classification. They can be extracted in a minimum bounding rectangle around the target to avoid interference of sea clutter. ${ }^{3}$ Hence, we measure the length and width of the deceptive target as critera for the comparison of the detection of classification performance. The measured length and width of the deceptive target simulated with the two methods are listed in Table 3. It is shown that the measured length and width of the two deceptive destroyers for the proposed method in various attitude are both similar to that of a destroyer represented in the 3-D model. However, there is an evident difference between the measured length and width of the deceptive targets in two attitudes for the method in Ref. 7. This is because it is impossible to represent a target in different attitudes using a 2-D deception-template model without changing the model. Therefore, the simulation results reveal that the proposed deception jamming method is able to generate the deceptive target in various attitude with higher fidelity by comparison.

Table 3 The measured length and width of deceptive target simulated with the two methods.

\begin{tabular}{lcccc}
\hline \hline \multirow{2}{*}{ Method } & \multicolumn{2}{c}{ Method in Ref. } & & \multicolumn{2}{c}{ Proposed method } \\
\cline { 5 - 6 } \cline { 5 - 6 } Target ID & DT1 & DT2 & DT3 & DT4 \\
\hline Length $(\mathrm{m})$ & 246.38 & 256.68 & 160.21 & 160.30 \\
Width $(\mathrm{m})$ & 37.35 & 43.69 & 20.07 & 20.71 \\
\hline \hline
\end{tabular}




\section{Conclusion}

In this paper, a target deception jamming method using the EM scattering is proposed, which counters the broadside and strip-map spaceborne SAR. In the proposed method, the modulation process is mainly divided into two stages: offline stage and real-time stage. In the offline stage, the proposed JFR is calculated by means of the EM scattering data from the deceptive target and further processing. In the real-time stage, the deception jamming is achieved by the DRFMbased SAR jammer system. Thus, the proposed method has a good prospect for practical application. Additionally, the EM scattering model can simulate the scattering characteristic of the target accurately, which is able to generate the deceptive target in various attitude with higher fidelity compared with the traditional deception jamming method. In conclusion, the simulation results of this paper show that the proposed method can generate well-focused false scatterers and generate the deceptive target in various attitude with high fidelity.

\section{Acknowledgments}

This work was supported in part by the National Natural Science Foundation of China (Grant No. 61771302) and the Aeronautical Science Foundation of China (Grant Nos. 20142057007 and 2015ZD07006).

\section{References}

1. I. Cumming and F. Wong, Digital Signal Processing of Synthetic Aperture Radar Data: Algorithms and Implementation, Artech House, Norwood, Massachusetts (2005).

2. W. Chao et al., "Ship detection for high-resolution SAR images based on feature analysis," IEEE Geosci. Remote Sens. Lett. 11(1), 119-123 (2014).

3. H. Lang et al., "Ship classification in SAR image by joint feature and classifier selection," IEEE Geosci. Remote Sens. Lett. 13(2), 212-216 (2015).

4. W. Walter and W. Goj, Synthetic Aperture Radar and Electronic Warfare, Artech House, Boston, Massachusetts (1993).

5. K. Dumper et al., "Spaceborne synthetic aperture radar and noise jamming," in Proc. IET Int. Radar System, pp. 411-414 (1997).

6. F. Zhou et al., "A novel method for adaptive SAR barrage jamming suppression," IEEE Geosci. Remote Sens. Lett. 9(2), 292-296 (2012).

7. F. Zhou et al., "A large scene deceptive jamming method for space-borne SAR," IEEE Trans. Geosci. Remote Sens. 51(8), 4486-4495 (2013).

8. Y. Liu et al., "A frequency-domain three-stage algorithm for active deception jamming against synthetic aperture radar," IET Radar Sonar Navig. 8(6), 639-646 (2014).

9. Q. Sun et al., "A novel jamming signal generation method for deceptive SAR jammer," in Radar Conf., pp. 1174-1178, IEEE (2014).

10. P. Pace et al., "Digital false-target image synthesiser for countering ISAR," IEE Proc. Radar Sonar Navig. 149(5), 248-257 (2002).

11. CST Studio Suite, CST Suite 2013, Computer Simulation Technology AG, Darmstadt (2013).

12. O. Dogan and M. Kartal, "Time domain SAR raw data simulation of distributed targets," EURASIP J. Adv. Signal Process. 2010(1), 784-815 (2010).

Qingyang Sun received his BS degree in electrical information engineering from Northeast University, China, in 2012. Since September 2012, he has been working toward his PhD at the Shanghai Key Laboratory of Intelligent Sensing and Recognition, Shanghai Jiao Tong University (SJTU), China. His research interests include radar signal processing, radar imaging processing, and radar countermeasure.

Ting Shu received his BS and MSc degrees in electronic engineering from Nanjing University of Science and Technology, China, in 2004 and 2006, respectively. Then, he received his PhD in electronic engineering from SJTU in 2010. Since 2011, he has been an assistant professor at 
the School of Electronic Information and Electrical Engineering (SEIEE), SJTU, China. His research interests include radar system design and implementation, radar signal processing, and radar countermeasure.

Bin Tang received his BS degree in communication engineering and his MSc degree in signal processing from Xidian University, China, in 1989 and 1997, respectively. Then, he received his $\mathrm{PhD}$ from SJTU in 2002. From 2002 to 2009, he was the fellow-class senior engineer at the Institute of Marine Electronic Equipment. Since 2009, he has been an associate professor at SEIEE, SJTU, China. His research interests include signal processing and radar countermeasure.

Wenxian Yu received his BS, MS, and $\mathrm{PhD}$ degrees from the National University of Defense Technology, Changsha, China, in 1985, 1988, and 1993, respectively. Currently, he is a professor and head of the SEIEE, SJTU. His research interests include target recognition, remote sensing information processing, multisensor data fusion, etc. In these areas, he has published more than 200 research papers. 